\title{
Intolerancia a los hidratos de carbono en la infancia
}

DR. ERNESTO GUIRALDES c. *

La intolerancia a los hidratos de carbono ha sido reconocida desde hace varias décadas como responsable directa o indirecta de la iniciación o prolongación de ciertas diarreas infantiles $(1,2)$. Con los recientes progresos en el campo de la fisiología de la absorción intestinal se ha clarificado notablemente el papel que les cabe a los hidratos de carbono tanto en la función normal como patológica del aparato digestivo.

Los carbohidratos representan la principal fuente energética del ser humano, y en el niño son de vital importancia por los requerimientos calóricos que el crecimiento le impone. En los primeros días de la vida el disacárido lactosa es el único azúcar de la dieta, situación que se modifica con la introducción de sacarosa y almidón, éste último en la forma de harinas. En grupos de bajos ingresos estos dos últimos tipos de hidratos de carbono constituyen una importantísima proporción de la dieta, hecho que debe ser tenido en consideración por sus implicaciones nutricionales y clínicas.

Numerosas revisiones del tema que nos preocupa han sido publicadas en la última década, y varias de ellas pueden servir de excelentes guías para el lector interesado en profundizar en la materia $(3-7)$.

\section{Definiciones y fisiología}

En diversas condiciones clínicas, la capacidad de la mucosa intestinal para hidrolizar los disacáridos está impedida, y es la lactosa la que más frecuentemente causa signos de intolerancia y anormalidad de los exámenes de laboratorio (3-7). Por tal razón su exclusión temporal de la dieta es la herramienta terapéutica más usada frente a estos cuadros. Menos frecuentemente la intoleran-

\footnotetext{
* Departamento de Pediatría, Sede Oriente, Facultad de Medicina, Hospital L. Calvo Mackenna.
}

cia se extiende a sacarosa y menos aún a los monosacáridos (3-6).

Debe distinguirse entre: "intolerancia a los disacáridos", que implica un concepto clínico, y: "deficiencia de disacaridasas" que expresa un substrato fisiopatológico no necesariamente traducible en síntomas y signos $(6,8)$. Es así como algunos pacientes con déficit de disacaridasas sólo experimentan diarrea y otros signos de intolerancia, al consumir disacáridos en cantidades que rebasen el umbral de acción de las enzimas citadas, por lo que sólo en este último caso puede hablarse de "intolerancia". Si en los mismos pacientes se realizan pruebas más finas de laboratorio (con cantidades menores de hidratos de carbono), puede que se detecte relativa mala absorción de ellos, pero sin que las concentraciones de azúcares no absorbidos en el lumen sean suficientes como para llevar a síntomas clínicos. Estas diferencias deben ser tomadas en cuenta para comprender cuándo y cómo un individuo es capaz de desarrollar un cuadro diarreico al ingerir hidratos de carbono.

Los carbohidratos de la dieta se pueden agrupar en tres categorías, de acuerdo al número de unidades básicas que los componen:

a) monosacáridos: compuestos que forman la unidad básica, la cual repetida un número variable de veces da origen al resto de los hidratos de carbono. En los alimentos de consumo humano no es frecuente encontrar cantidades apreciables de monosacáridos libres.

b) disacáridos: formados por la unión de dos monosacáridos y de los cuales los más frecuentemente encontrados en la dieta son: lactosa (glucosa-galactosa), sacarosa (glucosa-fructosa) y maltosa (glucosa-glucosa), ésta última como producto de la digestión intraluminal del almidón. 
c) Polisacáridos: resultantes de la unión, en número variable, de unidades de monosacáridos. El almidón y el g’icógeno, dos polisacáridos de alto peso molecular son los dos compuestos más representativos de este grupo.

Las amilasas, salival y pancreática, se encargan de la hidrólisis del almidón que, de esta manera al llegar al intestino delgado ha quedado reducido a mo'éculas más pequeñas, generalmente de dos a tres unidades de monosacáridos (disacáridos y trisacáridos, respectivamente) (9). Es importante consignar que de esta etapa de la hidrólisis de los polisacáridos no resultan monosacáridos como productos finales (9). Las protagonistas del siguiente paso son las disacaridasas, enzimas ubicadas $€ n$ la parte más apical del las células del epitelio absortivo intestinal (10). Como su nombre lo dice estas enzimas son hidrolasas que catalizan el desdoblamiento de los disacáridos a sus unidades constituyentes (monosacáridos). El hecho de estar situadas tan cerca del lumen intestinal las hace especialmente vulnerables a sufrir los efectos de diversas noxas, como se podrá apreciar más adelante. Las disacaridasas más importantes por su trascendencia clínica y nutricional son las siguientes (11):

a) lactasa: situada en el ápice de las microvellosidades de la célula epitelial intestinal y que cataliza la hidrólisis del disacárido lactosa.

b) sucrasa (o sacarasa): enzima que se encarga de la hidrólisis de sacarasa y de parte de la maltosa de la dieta.

c) isomaltasa: cuyos substratos son: isomaltosa, maltosa y algunas dæxtrinas límites (oligosacáridos).

d) Maltasa: que hidroliza la maltosa.

De esta manera, y como resultantes finales de las etapas hidrolíticas anteriormente citadas, en el lumen del duodeno y yeyuno habrá una cantidad apreciable de moléculas de monosacáridos. La glucosa es transportada al interior de la célufa en contra de su gradiente de concentración y a través de un mecanismo que utiliza energía celular, que es por lo tanto considerado como transporte activo (12). La energía no es ocupada directamente en incorporar la glucosa a la célula sino más bien en drenar el sodio intracelular hacia los espacios laterales y basales de la célula. De esta manera, el sodio del lumen entraría al enterocito a favor dz su propia gradiente de concentración, hecho que permite a la glucosa penetrar a la célula "acoplada" al transportador de membrana que transporta al sodio. Esta hipótesis, propuesta por Crane $(12,13)$ sigue siendo la más aceptada para explicar la sucesión de fe- nómenos que resultan eventualmente en incorporación de glucosa a la célula del epitelio intestinal. La glucosa y el sodio se potencian mutuamente en su entrada a la célula, por lo que la ausencia de uno de ellos en el lumen, disminuirá la eficiencia de la absorción del otro (12-14). En el yeyuno, el agua y otras moléculas pequeñas como cloro y potasio siguen al sodio y glucosa al interior de la célula mediante lo que se ha denominado fenómeno de "arrastre" $(15,16)$, que tiende a igualar las presiones osmóticas entre cl lumen intestinal y el espacio extracelular. El papel que se ha descrito para la glucosa puede ser asumido también por la galactosa (12) y algunos aminoácidos (17), que serían así competidores de la glucosa en cuanto a su absorción por la mucosa intestinal.

Es de interés agregar que algunos autores piensan actualmente que parte del transporte de glucosa en el intestino delgado pudiera ser realizado en forma pasiva a través de los espacios intercelulares: es decir sería un proceso extracelular (18).

Con respecto a la fructosa, durante mucho tiempo se pensó que su transporte era pasivo o a través de un sistema de absorción facilitada. pero actualmente hay evidencia en favor de un proceso activo $(19,20)$.

\section{Malabsorción de hidratos de carbono:}

Las causas de este sindrome son variadas, siendo ellas susceptibles de ocurrir simultáneamente en un mismo paciente. El caso de los lactantes desnutridos que sufren infecciones del tracto digestivo ilustra esta aserción, además de tener importancia epidsmiológica puesto que la malabsorción de hidratos de carbono puede contribuir a agravar una desnutrición subyacente.

En la tabla I se ha enumerado algunas de las etiologías que pueden llevar a una deficiente absorción de los azúcares de la dieta.

\section{I) Déficit de disacaridasas:}

Enfermedad Celiaca: La atrofia de las vellosidades intestinales es responsable de que los niveles de disacaridasas presentes en el enterocito, en esta enf:rmedad, estén muy reducidos, especialmente en el intestino delgado proximal (8). Sin embargo el hallazgo clínico de intolerancia a los hidratos de carbono en pacientes celíacos no es muy frecuente, ya que depende, como se ha dicho de la cantidad de azúcares ingerida. Si ésta se mantiene dentro de límites razonables podrá ser totalmente hidrolizada aun por las concentraciones relativamente bajas de disacaridasas presen- 
T A B L A I

CODICIONES ASOCIADAS CON INTOLERANCIA A HIDRATOS DE CARBONO

\section{Disacáridos:}

- Sindrome diarreico agudo o prolongado del lactante:

Diarrea infecciosa

Diarrea no específica

- Enfermedad Celíaca

- Cirugía intestinal en R.N. y lactantes menores

- Giardiasis masiva

- Desnutrición, especialmente proteica

- Deficiencia congénita de lactasa

- Deficiencia adquirida de lactasa, "racial" (especialmente en adultos).

- Deficiencia congénita de sucrasa-isomaltasa.

- Otras.

Colitis ulcerosa, Enfermedad de Crohn, Sprue Tropical.

Monosacáridos:

- Sindrome diarreico agudo severo en R.N. y en lactantes desnutridos.

- Cirugía intestinal en R.N. y lactantes menores.

- Anoxia severa neonatal.

- Infecciones graves extradigestivas en lactantes menores y R.N.

- Sindrome de "intestino delgado contaminado".

- Malabsorción congénita de glucosa-galactosa.

tes, sin llegar a producirse diarrea. Aún más, el compromiso enzimático es poco marcado en la parte distal del yeyuno y en el íleon $(6,8)$, que en este caso juegan un papel compensador. Estos hechos enfatizan la diferencia existente entre el hallazgo aislado de niveles bajos de disacaridasas en una diminuta muestra de biopsia intestinal y sus eventuales traducciones clínicas (6, 8).

\section{Gastroenteritis bacteriana o viral:}

En los últimos años, y gracias al empleo de biopsia intestinal en pacientes con gastroenteritis (algunos de ellos voluntarios sometidos al contagio de agentes bacterianos o virales) se ha podido demostrar que una alta proporción de estos cuadros diarreicos cursan con atrofia de las vellosidades intestinales, de grado variable $(8,21,24)$.

Tal atrofia no es pareja; por el contrario, pue- den existir zonas de mucosa profundamente alteradas al lado de otras relativamente indemnes (24). Por esta última razón no siempre existe traducción clínica de la deficiente actividad disacaridásica en algunas zonas, resultante del daño histológico de la mucosa. La medida clásica de diluir o suprimir temporalmente la leche (y otros alimentos que contienen azúcares) en los pacientes aquejados de gastroenteritis tiene su explicación fisiopatológica en la anormalidad arriba descrita $y$ en el hecho de que la mucosa intestinal suele recuperarse paulatina pero totalmente en el curso de pocos días (23). Sin embargo hay pacientes en los cuales la recuperación clínica tiene lugar en un tiempo bastante más prolongado $(6,8,25$ 27). A este grupo pertenecen los recién nacidos y los lactantes desnutridos, quienes ya tienen una capacidad funcional intestinal disminuida $(26,28)$ y son, por consiguicnte, más susceptibles de presentar diarreas prolongadas y complicadas (29$37)$, que a veces tienen un desenlace fatal (27, $33,34,36,38$ ).

La persistencia de deposiciones diarreicas en pacientes que han sufrido una gastroenteritis ("sindrome post-gastroenteritis") suele tener su explicación en una intolerancia transitoria a lactosa (y a veces a otros disacáridos) que se prolonga más allá de lo habitual. Como sustrato de esta condición se encuentra anormalidades de la mucosa intestinal tanto histológicas como bioquímicas (6, $25)$. Esta intolerancia a los azúcares es por lo tanto la responsable de un buen número de diarreas prolongadas en pacientes que vemos en hospitales y consultorios y debe ser sospechada en estos casos porque su corrección se obtiene al eliminar transitoriamente de la dieta los hidratos de carbono implicados. Junto con las anormalidades de la absorción de hidratos de carbono, $\mathrm{cn}$ el "sindrome post-gastroenteritis" pueden coexistir otras fallas de absorción como son las de las grasas y proteínas, de menor traducción clínica habitualmente, pero de implicancia nutricional que merece ser considerada.

\section{Enteritis difusas:}

A este grupo pertenecen entidades como la colitis ulcerosa (7) la Enfermedad de Crohn (39) y la enteritis secundaria a irradiación abdominal (40), que desembocan en malabsorción de hidratos de carbono a través de daño extenso de la mucosa intestinal.

\section{Lambiasis masiva:}

El hallazgo de intolerancia transitoria a leche es de relativa frecuencia en pacientes con infes- 
tación por g. lamblia $(41,42)$ pero el papel del parásito en esta condición no ha sido enteramente documentado hasta ahora, por lo que debe ser tomado con ciertas reservas.

\section{Resección intestinal extensa:}

La disminución de la superficic absortiva útil que ocurre en este tipo de cirugía lleva a intolerancia -a vices grave- a los azúcares de la dieta $(43,44)$, con importante repercusión en el estado nutritivo del paciente. Como estos enfermos también muestran variados grados da $a b-$ sorción defectuosa de otros nutrientes (44), hecho que coopera en la producción de diarrea, se comprend rá la importancia de su tratamiento oportuno con regímenes dietéticos apropiados.

\section{Cirugía abdominal en recién \\ nacidos y lactantes menores:}

El hallazgo de diarreas prolongadas luego de diversos tipos da cirugía abdominal en recién nacidos y lactantes menores (45) tendría su substrato fisiopatológico en una lesión del enterocito provocada por anoxia durante el acto quirúrgico, en una infección ent:ral agregada o en la combinación de ambas condiciones. El hecho de que causas no quirúrgicas que llevan a anoxia podrían también desencadenar una intolerancia a los hidratos de carbono (46), rispalda la primera de las hipótesis nombradas. En cuanto a la segunda, es apoyada por la demostración de proliferación de bacterias enteropatógenas en asas intestinales disvitalizadas como consecuencia de cirugía intestinal (45). Con frecuencia el defecto de absorción se extiende a todos los disacáridos así como a los monosacáridos, por lo que el pronóstico se ensombrec: y el tratamiento se dificulta considerablemente $(45)$.

\section{Desnutrición:}

Diferentes autores han informado de defectos en la hidrólisis de disacáridos en niños desnutridos, así como de falla en los mecanismos de transporte de los monosacáridos $(6,26,28,29,47)$. Este hecho parece ser especialmente prevalente en el tipo de desnutrición conocida como "desnutrición pluricarencial" o "Kwashiorkor" (26, 29,48 ), entidad que puede cursar con importante atrofia de las vellosidades intestinales (49, 50, 51). Otros mecanismos, como irfecciones bacterianas, virales y micóticas, e infestaciones parasitarias $(52,53)$, todas ellas frecuentes en la desnutrición, pueden agravar aún más el defecto básico de la mucosa. Más adelante, al hablar de "intestino delgado contaminado" se analizará con más detalle los mecanismos por los cuales las bacterias pueden llevar a mala absorción de hidratos de carbono.

\section{Ausencia congénita de disacaridasas:}

Dos entidades han sido reconocidas en este grupo: la ausencia de sucrasa-isomaltasa (54-57) y la alactasia o ausencia de lactasa (58). Los síntomas c'ínicos son severos y empiezan desde el momento en que el sujeto es enfrentado a los disacáridos correspondientes, que en el caso de la alactasia es el primero o segundo día de vida. Aun cuando la disacaridasa respectiva persiste ausente de la mucosa intistinal durante toda la vida del individuo, las manifestaciones clínicas parecen ir disminuyendo en intensidad con el tiempo (6), hecho que permite a los afectados tolerar pequeñas cantidades de alimentos que contengan los disacáridos implicados.

Ambas condiciones son muy raras, especialmente la ausencia congénita de lactasa $y$, en nuestro medio no hemos tenido conocimiento que hayan sido descritas hasta el momento.

\section{Intolerancia adquirida (racial) a lactosa:}

Esta condición, común a muchos adultos de diversos grupos étnicos en el mundo $(59,62)$ pudicra ya ser adquirida a temprana edad $(6,59)$. En este sentido se discute el papel que jugarían la desnutrición y las infestaciones e infecciones que afcetan a grupos infantiles de países en desarrollo $(6,61,63)$. La prevalencia geográfica de la intolerancia adquirida a lactosa, y las hipotesis que se han esgrimido para explicarla, están más allá del campo de esta visión, y al lector interesado $\mathrm{cn}$ el tema se le recomienda la lectura de algunos completos trabajos aparecidos en los últimos años $(60,62,64,66)$. Es interesante recalcar que en este contexto, y por razones de frecuencia, el adulto incapaz de tolerar lactosa pasaría a ser el "normal" mientras que el tolerante al disacárido sería la excepción (62).

\section{II) Malabsorción de monosacáridos:}

Muchas de las causas que llevan a malabsorción de monosacáridos son las mismas que producen déficit de disacaridasas, pero más acentuadas. Este hecho es comprensible si se tiene en cuenta que la célula epitelial intestinal responde en grado variable a la agresión, dependiendo del tipo, intensidad y extensión de ésta. Así, algunas patologías que afectan profundamente al enterocito pueden determinar tales alteraciones estruc- 
turales y bioquímicas en él, que eventualmente le impiden transportar adecuadamente los monosacáridos y otras moléculas pequeñas como agua y electrolitos $(67,68)$.

Algunos de los mecanismos que producen intolerancia a los monosacáridos y que merecen comentario especial son:

\section{Intestino delgado contaminado:}

Esta denominación $(67,69)$ es preferible a la anteriormente en boga de "asa ciega", ya que se aplica a cuadros no necesariamente producidos por anormalidades anatómicas del intestino delgado.

En diversos tipos de oclusión incompleta del lumen intestinal (42) o de existencia de cavidades no incorporadas al peristaltismo normal del intestino tiene lugar proliferación anormal de flora intestinal (42), que en condiciones habituales no coloniza segmentos tan altos del tubo digestivo. También puede encontrarse pululación anormal de distintos microorganismos en sujetos afectados de ciertas inmunodeficiencias celulares $(53,70)$, así como en desnutridos que viven en ambientes insalubros $(36,52)$. La acción bacteriana en estos casos puede traducirse en desconjugación y deshidroxilación de las sales biliares presentes en el intestino delgado alto $(71,73)$, induciendo la presencia de concentraciones anormalmente altas de sales biliares desconjugadas en el lumen de duodeno y yeyuno. Ha sido demostrado recientemente que tales sales biliares desconjugadas ejercen una acción tóxica sobre la mucosa del intestino delgado al ser depresoras de la actividad de la ATP-asa dependiente de sodio y potasio de la célula epitelial intestinal $(74,75)$. Dicha enzima cataliza la hidrólisis de ATP de la célula, proporcionando así la energía para que tenga lugar el transporte acoplado de sodio y glucosa que se describió más arriba. Así, puede comprenderse que, indirectamente, las bacterias pueden llevar a defectos en el transporte de monosacáridos ( $y$ de agua y electrolitos) a través de alteraciones inducidas sobre la estructura molecular de las sales biliares. Este mecanismo ha sido invocado, por lo tanto, como responsable de la intolerancia a monosacáridos que puede observarse en desnutridos con invasión bacteriana del intestino delgado $(6$, $36,53)$, siendo probable que en este grupo de pacientes operen simultáneamente múltiples factores interrelacionados $(6,76)$.

\section{Malabsorción congénita \\ de glucosa y galactosa}

Es una entidad extremadamente rara (77-79) que imposibilita a quienes la sufren, de tolerar cualquier alimento que contenga los monosacáridos arriba citados, por lo que estos pacientes deben ser tratados con dietas sumamente rigurosas para poder sobrevivir la primera infancia, ya que el defecto parece aminorarse en el curso de la vida (80-81). Esto último es válido sólo desde el punto de vista de la diarrea y otras manifestaciones clínicas puesto que la alteración funcional del enterocito jamás regresa $(80-81)$. Esta enfermedad constituye una "prueba de laboratorio" de la existencia de un sistema de transporte común para glucosa y galactosa, ya que ambos monosacáridos son afectados simultáneamente y jamás se ha descrito algún defecto de absorción de uno solo de ellos. Tampoco este error congénito ha sido descrito hasta ahora en Chile.

\section{Cirugía abdominal en recién nacidos $y$ lactantes menores:}

Como fuera explicado previamente, esta condición pucde llevar a intolerancia a disacáridos, pero en ocasiones es capaz también de extenderse a los monosacáridos (45), con lo que su tratamiento dietético se hace extremadamente complicado al requerir de fórmulas muy excluyentes, y su evolución clínica se agrava notablemente.

\section{Infecciones extradigestivas graves:}

Algunos de los casos descritos en la literatura, así como de nuestra propia experiencia, han sido de pacientes muy pequeños con infección grave, especialmente estafilocócica, del árbol respiratorio o de otros parénquimas (33-82). La patogenia de la malabsorción en estos casos no ha sido totalmente aclarada pero constituye un interesante ejemplo de la denominada diarrea "parenteral". Datos experimentales recientes (83) referentes a estudios efectuados en adultos, demuestran cierta interferencia en la absorción intestinal de algunos monosacáridos en infecciones sistémicas. Una extensión de dichos estudios a niños con determinadas infecciones extradigestivas permitirá aclarar el papel de ellas en la malabsorción de diversos nutrientes con lo que la diarrea parenteral dejaría de ser una condición todavía misteriosa.

En general, la malabsorción temporal de monosacáridos se ve en lactantes menores o recién nacidos, gravemente enfermos y constituye una complicación potencialmente letal, por llevar a hipoglicemia y a acidosis metabólica $(33,45)$. En nuestra experiencia, constituye un índice de mal pronóstico tanto por las etiologías que la provocan como por las complicaciones metabólicas aludidas. 
Mecanismos de producción de diarrea:

El mecanismo más importante que lleva a diarrea ante malabsorción de hidratos de carbono parece ser el osmótico (84-86). Al permanecer en el lumen intestinal concentraciones apreciables de disacáridos no digeridos (o monosacáridos no absorbidos) se produce atracción de agua que va a fluir desde la mucosa hacia el lumen. Este efecto resulta de la acción osmótica que ejercen las moléculas de pequeño tamaño no digeridas que en gran número ocupan la luz del intestino $(3,66$, 86) y tiene lugar hasta que las presiones osmóticas intra y extraluminal se igualan. Al progresar estos hidratos de carbono hacia el intestino grueso, a velocidad generalmente acelerada, debido al estímulo que sobre el peristaltismo produce el exceso de fluido $\mathrm{c} n$ el lumen $(3,85)$, tiene lugar una progresiva fermentación de los disacáridos, generada por la flora sacarolítica del colon (86). Las bacterias pueden hidrolizar a los disacáridos hacia sus monosacáridos constituyentes (87) y a éstos hacia ácidos orgánicos como láctico y pirúvico (88), e incluso hacia $\mathrm{CO}_{2}$ y agua $(6,66)$. De esta manera en el fluido del colon pueden coexistir disacáridos no digeridos, monosacáridos, ácidos orgánicos y anhidrido carbónico, ya quz rara vez la fermentación es total. Este hecho reviste importancia en el diagnóstico de intolerancia a los azúcares mediante el examen químico de las heces.

$\mathrm{El}$ que estos mecanismos anormales resulten o no en diarrea, dependerá en gran parte de la capacidad compensatoria del colon, ya que si éste puede absorbcr el fluido en exceso que se le ofrece desde el intestino delgado la diarrea será poco probable que se produzca (89). Pero en lactantes y en la infancia en general, el colon no tiene la capacidad suficiente como para compensar; de ahí que generalmente ocurra diarrea (6). Se ha invocado también que los ácidos orgánicos pudieran causar irritación química a las paredes del intestino (90) y que actuaran también osmóticamente cooperando así a la producción de diarrea (3). Estos dos últimos mecanismos parecen ser, en todo caso, menos importantes que el citado anteriormente.

Finalmente, restarían otros dos mecanismos que cooperarían a la diarrea existente. Uno es el papel de "caldo de cultivo" que los hidratos de carbono no digeridos tendrían sobre ciertos gérmenes del lumen intestinal (91), lo que llevaría a proliferación excesiva de ellos. El otro mecanismo se desprende de la fisiología y de la fisiopatología del transporte acoplado de monosacáridos, agua y clectrolitos, anteriormente explicado. Al no haber una cantidad suficiente de glucosa y galactosa que sean transportadas al interior de la célula, bajará también la eficiencia del trans- porte intestinal de agua y electrolitos, con lo que éstos compuestos permanecerán en el lumen en cantidad superior a la habitual y contribuirán así a la diarrea.

\section{Características clinicas y diagnóstico de la malabsorción de hidratos de carbono:}

Debido a la presencia de ácidos orgánicos en las deposiciones, éstas son de olor ácido, irritantes de la piel de la zona giútzo-genital, y de un $\mathrm{pH}$ generalmnte inferior a 5.5. Son además líquidas (por el efecto osmótico de los azúcares no digcridos o no absorbidos), explosivas (por el $\mathrm{CO}_{2}$ que contienen) y son precedidas por dolores cólicos, borborigmos y distensión abdominal.

En ocasiones existe lactosuria, derivada de la climinación renal de moléculas de lactosa que, intactas atraviesan la pared intestinal cuya permeabilidad está alterada $(3,66)$.

Los lactantes con severa intolerancia a los hidratos de carbono se nos presentan a menudo camo pacientes con intensa deshidratación y acidosis, refractarios a los tratamientos convencionales.

En los párrafos anteriores ha habido referencias a algunas características que permiten sospechar la intolerancia a los azúcares en algunos pacientes. Además de una cuidadosa historia clínica, con énfasis en los aspectos dietéticos, existen varias pruebas que pueden confirmar el diagnóstico.

Hay un grupo de exámenes que se basan en la presencia en las heces de cantidades anormales de sustancias reductoras (a las cuales pertenecen todos los monosacáridos y disacáridos, con excepción de la sacarosa). En este grupo de pruebas se incluyen: a) Clinitest (87), ampliamente usado en distintos lugares y frecuentemente descrito en la literatura, b) Fehling y c) Benedict. Estos dos últimos han sido de gran aceptación en nuestro medio $(92,93)$ así como en otros lugares (76), tanto por su bajo costo como por su simple ejecución. Estas dos cualidades hacen que estas pruebas sean especialmente útiles en un medio como el nuestro, con tan alta incidencia de diarreas infantiles.

La Cromatografía en papel permite identificar cualitativamente los azúcares presentes en las deposiciones (94), pero su uso está restringido a la investigación de casos individuales y no a la práctica de rutina.

Es importante recalcar que cualquier examen de los arriba citados requiere hacerse en la fracción líquida de las deposiciones la cual debe ser obtenida con la ayuda de una sonda rectal o con un dedo enguantado. La deposición no debe ser tomada del pañal puesto que éste absorbe el agua 
que contiene, y con ella, las sustancias solubles presentes. También es importante realizar las prue bas de laboratorio en heces frescas o mantenidas bajo congelación, ya que la temperatura ambiental puede acelerar la fermentación y entregar resultados falsamente negativos.

La presencia de ácidos orgánicos en las deposiciones otorga a éstas la propiedad de tener un $\mathrm{pH}$ que generalmente es menor de 5.5. La correlación entre substancias reductoras positivas $y$ $\mathrm{pH}$ bajo se encuentra con frecuencia relativamente alta en nuestra experiencia $(93,95)$, pero la discordancia entre ambas pruebas no debe ser razón para descartar el diagnóstico. Es así como, la ausencia de flora sacarolítica en pacientes que han recibido ciertos antibióticos puede hacer que, habiendo exceso de substancias reductoras en las deposiciones, el pH sea de 6 ó más $(27,93)$, debido a que no ha existido suficiente fermentación hacia ácidos orgánicos. Por el contrario, un $\mathrm{pH}$ bajo con substancias reductoras negativas puede verse en las primeras etapas de la intolerancia a los hidratos de carbono (84) o en algunos casos de intolerancia sacarosa (95) y debe obligar a que el examen sea repetido. La hidrólisis previa de las deposiciones con $\mathrm{HCl} 1 \mathrm{~N}$ aclara este último diagnóstico al escindir la sacarosa a glucosa y fructosa, ambas con propiedades reductoras (3, 95). El uso de cinta $\mathrm{pH}$ pudiera ocasionalmente ofrecer resultados ambiguos e incluso discrepantes con la medición simultánea del $\mathrm{pH}$ en un $\mathrm{pH}$-ímetro con electrodo de vidrio (95), por lo que se recomienda el uso alternativo de este instrumento en los casos dudosos. La medición cuantitativa de ácido láctico en heces (92) es un excelente medio de aclarar diagnósticos dudosos, por su fidelidad, aunque resulta largo y engorroso, y poco apropiado por lo tanto como prueba de rutina para nuestro medio.

La determinación directa de actividad disaca. ridásica en un trozo de biopsia intestinal puede constituir una prueba necesaria con fines de investigación o en casos sospechosos de presentar ausencia congénita de disacaridasas, pero lógicamente, no tiene lugar dentro de la práctica diaria (6). Mayor utilidad ofrecen las curvas de tolerancia oral a los azúcares sospechosos de ser anormalmente absorbidos. Sin embargo, debe tenerse en cuenta que las curvas de absorción, medidas como glicemia, pueden estar alteradas en condiciones ajenas a la malabsorción (90), y que habitualmente resulta de mayor provecho diagnóstico la existencia de síntomas clínicos digestivos provocados por el examen (3).

Finalmente, el diagnóstico definitivo de intolerancia a los azúcares suele estar dado por la respuesta clínica a la supresión del azúcar sospechoso de la dieta, y también por la recaída secundaria a su reintroducción prematura (89).

\section{Tratamientos:}

El tratamiento lógico de la intolerancia a los hidratos de carbono debe ser la supresión del o de los azúcares causantes. Así, se comienza suprimiendo la lactosa de la dieta y reemplazándola por sacarosa o maltosa, disacáridos ambos menos comprometidos en su absorción intestinal. $\mathrm{Si}$ el paciente persiste con diarrea y hubiera aún eliminación de susbtancias reductoras por las deposiciones, el siguiente paso debe consistir en el reemplazo de los disacáridos citados por los monosacáridos glucosa y fructosa. El primero de éstos es el más usado, por ser de más fácil obtención, aunque parece ser que su absorción se ve comprometida más frecuentemente que la de fructosa $(31,82,96)$. Desde un punto de vista práctico, para la intolerancia a lactosa, en nuestros hospitales se usan habitualmnte mezclas consistentes en carne de vacuno o de pollo, féculas de papa o arroz, aceite y sacarosa y/o maltosa. Dichas mezclas tienen el inconveniente de carecer de una buena parte de los minerales y vitaminas que caracterizan una alimentación infantil equilibrada, por lo que estos nutrientes deben ser proporcionados a los pacientes en forma suplementaria (82). La duración del tratamiento por tal motivo, no debiera sobrepasar un tiempo razonable para no llevar a los enfermos a problemas carenciales. En este momento, aún no disponemos en nuestro país de alimentos industriales sin disacáridos, o sin carbohidratos, pero es probable que esta ausencia sea subsanada en el futuro próximo. En casos individuales (97) o con fines de investigación se ha usado con éxito leche tratada con la enzima lactasa, pero este tratamiento tiene el inconveniente de su carestía que impide su aplicación habitual en la práctica clínica.

Otras fórmulas alimenticias, complejas pero susceptibles de ser preparadas en algunos hospitales, también han sido recomendadas por algunos autores (82).

En nuestro hospital el esquema usado consiste en indicar una mezcla sin lactosa en cuanto se documenta mala absorción de hidratos de carbono en un paciente que presente diarrea. Si éste no manifiesta intolerancia a otros carbohidratos, a los siete días más o menos se intenta la reintroducción gradual de leche en la dieta, si es que la diarrea ya ha cedido. Cuando el enfermo aún no está en condiciones de tolerar leche se producirá diarrea de $\mathrm{pH}$ ácido y con exceso de sustancias reductoras, por lo que la fórmula sin leche deberá ser mantenida por otro período. El mismo esquema es aplicable a la mala absorción combinada de lactosa y otros azúcares, caso en el que se usará alguna mezcla alternativa de las descritas más arriba. 
T A B L A I I

CAUSAS DE FRACASO REAL O APARENTE

EN EL USO DE MEZCLAS SIN LACTOSA

Persistencia de la diarrea:

- Administración de una fórmula con disacáridos, por error.

- Uso de medicamentos que contengan lactosa como excipiente.

- Malabsorción asociada de otros hidratos de carbono.

- Diarrea infecciosa severa subyacente.

- Enfermedad Celíaca no tratada con exclusión del gluten.

\section{Curva ponderal plana:}

- Desnutridos en el período estacionario ponderal.

- No suplementación de la dieta con los minerales y vitaminas necesarios.

- Uso de mezclas con excesiva concentración de proteínas.

Cada vez que se esté en presencia de una mala absorción de hidratos de carbono hay que plantearse la utilidad que se obtendrá al suprimir de la dieta los azúcares causantes, y si ésta será la única medida terapéutica. En caso de condicio nes clínicas como Enfermedad Celíaca, reserción intestinal, "sindrome postgastroenteritis" y otras, indudablemente la supresión de los azúcares no absorbidos redundará en provecho para el enfermo, puesto que la mala absorción de ellos juega un papal patogénico decisivo en la producción de diarrea, aunque no sea el único mecanismo que lleva a ella. Pero, en algunas enteritis graves infecciosas pucden coexistir tantos mecanismos en la génesis de la diarrea (98), que la mera supresión de los hidratos de carbono de la dieta no provocará beneficio visible. Estos pacientes suelen tener un peristaltismo tan acelerado que los nutrientes no alcanzan a tomar contacto con las paredes intestinales como para ser digeridos o absorbidos en su totalidad, siendo así eliminados en exceso por las deposiciones. Por tal razón, las medidas terapéuticas deben ser acordes con la enfermedad básica y tomar en consideración la gran expoliación de agua y electrolitos a que están expuestos dichos pacientes. Enfermedades que ejemplifican el caso aludido son el cólera y algunas diarreas por E. Coli Enteropatógena.

Además de los ejemplos anotados hay otras condiciones en que el tratamiento fracasa, y ellas son enumeradas en la tabla 2.
Finalmente, hay un grupo de pacientes entre los que destacan recién nacidos y lactantes menores extremadamente desnutridos o portadores de patología digestiva catastrófica, cuya refractariedad al tratamiento ha hecho plantear a muchos autores el empleo de medidas "heroicas" para lograr sacarlos adelante. Hoy en día, la alimentación intravenosa tiene un papel preponderante en este sentido (99) y es necesario preparar en nuestro medio las condiciones para que sea puesta en funciones en forma regular. Otras medidas preconizadas $(37,100)$ pueden también tener su papel en nuestros esquemas terapéuticos y debieran ser evaluadas para emplearlas cuando la ocasión así lo requiera.

\section{REFERENCIAS}

1.- Finkelstein, H.: En "Tratado de las enfermedades de los niños de pecho". Tercera Edición. Ed. Labor, 1924.

2.-Howland, J.: "Prolonged intolerance to carbohydrates". Trans. Am Pediatr. Soc. 53: 11, 1921.

3.-Townley, $H .:$ "Disaccharidase deficiency in infancy and childhood". Pediatrics 39: 127, 1966. 1966.

4.- Anderson, C. M.: "Disorders of carbohydrate absorption in childhood" J. Clin. Pathol. (Suplemento) $24: 14,1971$.

5.- Holzel, A.: "Sugar malabsorption duc to deficiencies of disaccharidase activities and of monosac charide transport". Arch. Dis. Childh. 42: 341, 1967.

6.- Gracey, M. y Burke, V.: "Sugar induced diarrhoea in children'. Arch. Dis. Childh. 48: 331, 1973.

7.--Gray, G. M.: Malabsorption of carbohydrate". Fed. Proc. 26: 145, 1967.

8.-Anderson, C. M.; Burke, V.; Messer, M. y Kerry, K. R.: "Sugar intolerance and coeliac Disease". Lancet 1: 1322, 1966.

9.-Gray, G. M.: "Carbohydrate digestion and absorption”. Gastroenterology 58: 96, 1970.

10.- Miller, D. y Grane, R. K.: "The digestive function of the epithelium of the small intestine. II Localization of disaccharide hydrolysis in the isolated brush border portion of intestinal epitehelial cells"' Biochim. Biophys. Acta 52: 293, 1961.

11.-Lindquist, B.: Malabsorption of carbohydrates: enzyme and transport abnormalities". Presentado al XIV Congreso Internacional de Pediatría, Buenos Aires, 1974.

12.-Crane, R. K.: "Absorption of sugars". Handbook of Physiology, vol. 3. Alimentary Canal, secc. 6, p. $1323,1968$.

13.- Crane, R. J.: "Hypothesis for mechanism of intestinal active transport of sugars'. Fed. Proc. 21: 891, 1962. 
14.-Schedl, H. P. y Clifton, J. A.: "Solute and water absorption by the human small intestine". Nature 199: 1264, 1963.

15.-Turnberg, L. A.; Fordtran, J. S.; Carter, N. W. y Rector, F. C. Jr.: "Mechanism of bicarbonate absorption and its relationship to sodium transport in the human jejunum". J. Clin Invest. 49: 548, 1970.

16.- Turnberg, L. A.: "Potassium transport in the human small bowell". Gut 12: 811, 1971.

17.-Fordtran, J. S.; Rector, F. C. jr. y Carter, N. W.: "The mechanisms of sodium absorption in the human small intestine". J. Clin. Invest. 47: 884, 1968.

18.- Parsons, D. S.: Información personal.

19.-MacRae, A. R. y Neudoerffer, T. S.: "Support for the existence of an active transport mechanism of fructose in the rat". Bichim. Biophys. Acta 288: 137, 1972.

20.-Gracey M., Burke, V. y Oshin, A.: "Active intestinal transport of D - fructose". Biochim. Biophys. Acta 266: 397, 1972.

21.- Toccalino, H., Licastro, R., Cardo, A. G. y Williams, U.: Histologic changes in the small intestine in children with acute diarrhea". J. Pediat. 78: 1971 (Extracto).

22.-Agus, S. G.; Dolin, R.; Wyatt, R. G.; Tousimis, A. J. y Northrup, R. S.: "Acute infectious nonbacterial gastroenteritis: intestinal histopathology". Ann. Intern. Med. 79: 18, 1973.

23.-Barnes, G. L. y Townley, R. R. W.: "Duodenal mucosal damage in 31 infants with gastroenteritis". Arch. Dis. Childh. 48: 343, 1973.

24.-Walker-Smith, J. y Rossiter, M.: "Abnormal mucosa in gastroenteritis". Arch. Dis. Childh. 48: 746, 1973.

25.-Burke, V.; Kerry, K. B. y Anderson, C. M.: "The relationship of dietary lactose to refractary diarrhea in infancy". Aust. Paed. J. 1: 147, 1965.

26.-James, W. P. T.: Jejunal disaccharidase activities in children with marasmus and with Kwashiorkor. Response to treatment". Arch. Dis. Childh. 46: $218,1971$.

27.-Lifshitz, F.; Coello-Ramírez, P.; Gutiérrez-Topete, G. y Cornado - Cornet, M. C.: "Carbohydrate intolerance in infants with diarrhea". J. Pediatr. 79: $760,1971$.

28.-James, W. P. T.: "Sugar absorption and intestinal motility in children when malnourished and after treatment". Clin. Sci. 39: 305, 1970.

29.-Bowie, M. D.; Brinkman, G. L. y Hansen, J. D. L.: "Acquired disaccharide intolerance in malnutrition". J. Ped. 66: 1085, 1965.

30.- Wharton, B.: Metabolic effects of malnutrition in childhood". J. Roy. Coll. Phycns. Lond. 7: 259, 1973.

31.- Wharton, B. A.: "Difficulties in the initial treatment of kwashiorkor". En: "Calorie deficiencies and Protein deficiencies". Edit. R. A. Mc Cance y E. M. Widdowson. J. A. Churchill Ltd. Londres, 1968.

32.-Danús, O.; Urbina, A. M.; Soriano, H.; Guerrero, P.; Macaya, J.; Campos, C. y Fernández, M. E.: "Intolerancia a lactosa en el sindrome diarreico agudo del lactante". Pediatría, (Santiago). 13: $51,1970$.

33.-Lifshitz, F.; Coello-Ramirez, P. y GutiérrezTopete G.: "Monosaccharide intolerance and hypoglicemia in infants with diarrhea. I Clinical course of 23 infants". J. Pediat. 77: 595, 1970.

34.-Lifshitz, F.; Coello-Ramírez, P. y Gutiérrez-Topete, G.: "Monosaccharide intolerance and hypoglicemia in infants with diarrhea. II. Metabolic studies in 23 infants." J. Pediatr. 77: 604, 1970.

35.- Walker, A. C. y Harry, J. G.: "A survey of diarrhoeal disease in malnourished aboriginal children". Med. J. Austr. 1: 904, 1972.

36.-Gracey, M. y Bower, G.: "Fatal diarroeal disease in Australian Aboriginal children". Aust. N. Z. J. Med. 3: 255, 1973.

37.-Graham, G. G.; Baertl. J. M.; Cordano, A. y Morales, E.: "Lactosefree, Medium - chain triglyceride formulas in severe malnutrition". Am. J. Dis. Child. 126: 330, 1973.

38.-Coello-Ramirez, P.; Gutiérrez-Topete, G. y Lifshitz, F.: "Pneumatosis intestinalis". Am. J. Dis. Child. 120: 3, 1970.

39.-Dyer, N. H. y Dawson, A. M.: "Malabsorption". Brit. Med. J. 2: 161, 1968.

40.--Bond, $V$.P.: "Effects of radiation on intestinal absorption". Am. J. Clin. Nutr. 12: 194, 1961.

41.-Durand, P.: "Lactose intolerance". En: "Disorders due to intestinal defective carbohydrate digestion and absorption". Ed. Durand, P., 1964.

42.- Kirsner, J. B.: "Clinical observations on malabsorption". Med. Clin. North Am. 53: 1169, 1969.

43.- Weijers, H. y Van de Kamer, J. H.: "Diarrhoea caused by deficiency of sugar splitting enzymes". II Acta Paediat (Scand) 51: 371, 1962.

44.-Anderson, C. M.: "Intestinal malabsorption in childhood". Arch. Dis. Childh. 41: 571, 1966.

45.- Burke, V. y Anderson, C. M.: Sugar intolerance as a cause of protracted diarrhoea following surgery of the gastrointestinal tract in neonates". Aust. Paediat. J. 2: 219, 1966.

46.-Akesode, F.; Lifshitz, F. y Hoffman, K. M.: "Transient monosaccharide intolerance in a newborn infant". Pediatrics 51: 891, 1973.

47.- James, W. P. T.; Drasar, B. S. y Miller, C.: "Physiological mechanisms of weanling diarrhea". Am. J. Clin. Nutr. 25: 564, 1972.

48.-Bowie, M. D.; Brinkman, G. L. y Hansen, J. D. L.: Diarrhoea in Proteincalorie malnutrition". Lancet 2: 550, 1963.

49.-Stanfield, J. P.; Hutt, M. S. R. y Tunnicliffe, R.: "Intestinal Biopsy in Kwashiorkor". Lancet 2: 519, 1965. 
50.-Tandon, B. N.; Magotra, M. L.; Saraya, A. K. y Ramalingaswami, $V .:$ "Small intestine in protein malnutrition”. Am J. Clin. Nutr. 21: 813, 1968.

51.-Brunser, O.; Reid, A.; Monckeberg, F.; Maccioni, A. y Contreras, 1.: "Jejunal mucosa in infant malnutrition”. Am. J. Clin. Nutr. 21: 976, 1968.

52.-Gracey, M. y Stone, D. E.; "Small intestinal microflora in Australian aboriginal children with chronic diarrohoea". Aust. N. Z. J. Med. 2. 215, 1972.

53.-Gracey, M.: "The effects of intestinal microorganisms on intestinal absorption". Presentado al XIV Congreso Internacional de Pediatría, Buenos Aires, 1974.

54.-Auricchio, S.; Rubino, A.; Prader, A.; Rey, J.; Jos., J.; Frezal, J. y Davidson, M.: “Intestinal glycosidase activities in congenital malabsorption of disaccharides". Pediat. 66: 555, 1965.

55.-Kerry, K. R. y Townley R. R. W.: "Genetic aspects of intestinal sucrase isomaltase deficiency". Aust. Paediat. J. 1: 223, 1965.

56.-Antonowicz, 1.; Lloyd-Still, J. D.; Khaw, K. T. у Schwachman, B.: "Congenital sucrase - isomaltase deficiency. Observations over a period of 6 years". Pediatrics 49: 847, 1972.

57.-Ament, M. E.; Perera, D. R. y Esther, L. J.: "Sucrase - isomaltase deficiency-a frequently misdiagnosed disease". J. Pediatr. 83: 721, 1973.

58.-Levin, B.; Abraham, J. M.; Burgess, E. A. y Wallis, P. G.: "Congenital lactose malabsorption". Arch. Dis. Childh 45: 173, 1970.

59.-Chung, M. H. y Mc. Gill, D. B.: "Lactase deficiency in orientals". Gastroenterology 54: 225, 1968.

60.-Kretchmer, N.: "Lactose and lactase - a historical perspective". Gastroenterology 61: 805, 1971.

61.-Bayless, T. M.; Paige, D. M. y Ferry, G. D.: "Lactose intolerance and milk drinking habits". Gastroenterology 60: 605, 1971.

62.-Simoons, F. J.: "New Light on ethnic differences in adult lactose intolerance". Amer. J. Digest. Dist. 18: 595, 1973.

63.-Cook, G. C. y Lee, F. D.: "The jejunum after Kwashiorkor". Lancet 2: 1263, 1966.

64.-Simoons, F. J.: "Primary adult lactose intolerance and the milking Habit: a problem in biological and cultural interrelations: I Review of the medical research. Amer. J. Digest. Dis. 14: 819, 1969.

65.- Idem, parte II.: "A culture historical hypothesis". Amer. J. Digest. Dis. 15: 695, 1970.

66.-Kretchmer, N.: "Lactose and lactase". Scientific American 227: 70, 1972.

67.-Gracey, M.: "Intestinal absorption in the contaminated smallbowel syndrome". Gut 12: 403, 1971.

68.-Harries, J. T. y Sladen, G. E.: "The effects of different bile salts on the absorption of fluid, electrolytes and monosaccharides in the small intestine of the rat in vivo". Gut. 13: 596, 1972.
69.- Challacombe, D. N.: "A study of the duodenal microflora and bile salts in the contaminated small syndrome". Presentado al Congreso de la Sociedad Británica de Pediatría, abril 1973, Lancáster.

70.-Waldman, T. A. y Laster, L.: "Abnormalities of albumin metabolism in patients with hypogammaglobulinemia". J. Clin. Invest. 43: 1025, 1964.

71.-Dietschy, J. M.: "Effects of bile salts on interdiate metabolism of the intestinal mucosa". Fed. Proc. 26: 1589, 1967.

72.-Donaldson, R. M. Jr.: "Role of enteric microorganisms in malabsorption". Fed. Proc. 26: 1426, 1967.

73.-Davidson, A. M.: "Bile salts and fat absorption". Gut 8: 1, 1967.

74.-Gracey, M.; Papadimitriou, J.; Burke, V.; Thomas, J. y Bower, G.: "Effects on small-intestinal function and structure induced by feeding a deconjugated bile salt". Gut 14: 519, 1973.

75.-Guiraldes, E.; Oyesiku, J. E.; Lamabadusuriya, S. P. y Harries, J. T.: "Selective inhibition of mucosal $\mathrm{Na}-\mathrm{K}$ adenosine Triphosphatase (A $\mathrm{T}$ Pase) by deoxycholate in the jejunum in vivo". Acta Paediat. Scand. 63: 662, 1974 (Extracto).

76.-Morley, D.: En Paediatric Priorities in the developing world". Capítulo 10. Editor John Apley, Butterworths Publishers, 1973.

77.-Lindquist B. y Meewise, G. W.: "Chronic diarrhoea caused by monosaccharide malabsorption". Acta Paediat, Scand. 51: 674, 1962.

78.-Anderson, C. M.; Kerry, K. R. y Tounley, R. R. W.: "An inborn deffect of interstinal absorption of certain monosaccharides". Arch. Dis. Childh. 40: $1,1965$.

79.- Wimberley, P. D.; Harries, J. T. y Burgess, E. A.: "Congenital glucosegalactose malabsorption". Proc. Roy. Soc. Med. 67: 25, 1974.

80.- Elsas, L. J. y Lambke, D. W.: "Familial glucosegalactose malabsorption. Remission of glucose intolerance"' J. Pediat. 83: 226, 1973.

81.-Phillips, S. F. y Mc. Gill, D. B.: "Glucose-galactose malabsorption in an adult: perfusion studies of sugar, electrolyte and water transport. Amer. J. Dig. Dis. 18: 1017, 1973.

82.-Harries, J. T. y Francis, D. E. M.: "Temporary monosaccharide introlerance". Acta Paediat Scand. 57: 505, 1968.

83.-- Cook, G. C.: "Some factors influencing absortion rates of the digestion products protein and carbohydrate from the proximal jejunum of man and their possible nutritional implications". Gut 15: 139, 1974.

84.-Torres - Pinedo, R.; Rivera, C. L. y Fernández, S.: "Studies on infant diarrhea. II Absorption of glucose and net fluxes of water and sodium chloride in a segment of the jejunum". J. Clin. Invest. 45: 1916, 1966. 
85.- Launiala, K.: "The effect of unabsorbed sucrose or mannitol - induced accelerated transit on absorption in the human small intestine" Scand. J. Gastroent. 4: 25, 1969.

86.- Christopher, N. L. y Bayless, T. M.: "Role of the small bowel and colon in lactose-induced diarrhea". Gastroenterology 60; 845, 1971.

87.- Kerry, K. R. y Anderson, C. M.: "A ward test for sugar in faeces". Lancet 1: 981, 1964.

88.- Weijers, H. A.; Van de Kamer, J. H.; Dicke, W. K. $y$ cols.: Diarrhoea caused by deficiency of sugar - splitting enzymes". I. Acta Paediat. (Scand) 50: 55, 1961.

89.-Bartrop, R. W. y Hull, D.: "Transiet lactose intolerance in infancy". Arch. Dis. Childh. 48: 963, 1973.

90.-Weijers, H. A. y Van de Kamer, J. H.: "Fermentative diarrhoeas". En: "Disorders due to intestinal defective carbohydrate digestion and absorption". Edit. Durand, P., Roma Il Pensiero Scientifico, 1964.

91.-- Coello-Ramirez, P. y Lifschitz, F.: "Enteric microflora and carbohydrate intolerance in infants with diarrhea". J. Pediat. 79: 760, 1971.

92.-- Soriano, H.; Macaya, J.; Duffau. G.; Garcia, 1. y Urbina, A. M.: "Sindrome diarreico agudo del lactante. Empleo de la reacción de Fehling en el diagnostico de la intolerancia a los disacáridos". Pediatría (Santiago) 15: 452, 1972.

93.-Guiraldes, E.: "Comparación entre diversos métodos para diagnóstico rápido de malabsorción de hidratos de carbono". Revista Chilena de Pediatría, en prensa.

94.-Soeparto, P.; Stobo, E. A. y Walker-Smith, J. A.: "Role of chcmical examination of the stool in diagnosis of sugar malabsorption in children" Arch. Dis. Childh. 47: 76, 1972.

95.-Hidalga, C.; Leiva, H.; Rodriguez, G.; Venegas C. G. y Guiraldes E.: Datos no publicados.

96.-Walker-Smith, J.; Paterson, H. y Keating, S.: "Evaluation of a new feeding formula for sugar malabsorption". Med. J. Austr. 2: 107, 1973.
97.-- Moynahan, E. J. y Barnes, P. M.: "Zinc deficiency and a synthetic diet for lactose intolerance". Lancet 1: 676, 1973.

98.-Grady, G. F. y Keusch, G. T. "Pathogenesis of bacterial diarrheas". New Engl. J. Med. 285: $83189,1971$.

99.-Lloyd-Still, J. D.; Schwachman, H. y Filler, R. M.: "Intravenous hiperalimentation in pediatrics". Amer. J. Digest. Dis. 17: 1043, 1972.

100.-Akram Tamer, M.; Santora, T. R. y Sandberg, D. H.: "Cholestyramine therapy for intractable diarrhea". Pediatrics 53: 217, 1974.

\section{ADDENDUM :}

Trabajos recientes (a, b, c, d, e), publicados después de finalizado este manuscrito, hacen importantes contribuciones a la experiencia nacional sobre intolerancia a los hidratos de carbono, especialmente en cuanto a su relación con el Sindrome diarreico agudo del lactante.

\section{REFERENCLAS}

a) Emilfork, M.; Duffau, G.; Macaya, J. y cols.: "Sindrome diarreico agudo con deshidratación del lactante. Estudio con metódica de balance de dos fórmulas dietéticas". Pediatría (Santiago) 17: 75, 1974.

b) Calderón, A.; Goldenberg, E.; Macaya, J. y cols.: "Sindrome diarreico agudo de evolución tórpida. Análisis clínico de 100 pacientes". Id. pág. 95.

c) Duffau, G.; Emilfork, M.; Macaya, J. y cols.: "Sin drome diarreico agudo de evolución tórpida del lactante. Intolerancia secundaria a sacarosa". Id. pág. 103.

d) Emilfork, M. y Duffau, G.: "Sindrome diarreico de evolución tórpida del lactante. Intolerancia secundaria a hidratos de carbono. Metódica de estudio". Id. pág. 107.

e) Macaya, J.; Duffau, G.; Soriano, H. y cols.: "Empleo de exámenes cualitativos junto a la cama del enfermo con sindrome diarreico agudo con deshidratación". Id. pág. 113. 